\title{
SHEPHERDING OF THE URANIAN RINGS. II. DYNAMICS
}

\author{
PETER GOLDREICH \\ Division of Geological and Planetary Sciences, California Institute of Technology, Pasadena, California 91125 \\ Carolyn C. Porco \\ Lunar and Planetary Laboratory, University of Arizona, Tucson, Arizona 85721 \\ Received 7 October 1986; revised 18 November 1986
}

\begin{abstract}
We explore the dynamical significance of the orbital resonances, identified in Paper I (Porco and Goldreich 1987), involving the satellites 1986U7 and 1986U8, and the $\epsilon, \delta$, and $\gamma$ rings. We demonstrate that these satellites are capable of exerting torques on the $\epsilon$ ring which supply at the inner edge, and remove at the outer edge, the unperturbed angular-momentum luminosity transported by viscous stresses outward across the ring, provided that the ring has a mass which is comparable to the predicted value $M_{\epsilon}=6.1 \times 10^{18} \mathrm{~g}$, and that it is not more than a few meters thick. Thus there is no compelling reason to question the applicability of the standard theory of shepherding to the $\epsilon$ ring. However, the standard theory does place rather stringent requirements on the ring's properties, suggesting that confinement might be due to the reduction of the angular-momentum luminosity by flux reversal. Flux reversal could be associated either with the ring's eccentricity gradient or with nonlinear density waves in its interior. The drag due to the planet's extended neutral hydrogen atmosphere probably has only a minor effect on the dynamics of the $\epsilon, \delta$, and $\gamma$ rings. However, it poses a severe problem for the shepherding of the $\alpha$ and $\beta$ rings unless their masses have been seriously underestimated. This problem, and the large $s$-band optical depths of these rings, lead us to question the proposal that self-gravity is responsible for enforcing rigid precession in narrow rings.
\end{abstract}

\section{INTRODUCTION}

The discovery of ten small satellites interior to the orbit of Miranda during the Voyager Uranus encounter (Smith et al. 1986) has given us the opportunity to test theoretical predictions of planetary ring dynamics. The results of an exhaustive search for resonant interactions between these ten new satellites and the Uranian rings was described in a companion paper (Porco and Goldreich 1987, hereafter referred to as Paper I). Several low-order resonances involving the two satellites straddling the $\epsilon$ ring, 1986U7 and 1986U8, and the $\gamma, \delta$, and $\epsilon$ rings were identified. Based on an examination of the kinematics of these resonances, we proposed that 1986U7 and 1986U8 are shepherding the $\epsilon$ ring, that 1986U7 is the outer shepherd for the $\delta$ ring, and that 1986U8 is an outer shepherd for the $\gamma$ ring. In this paper, we apply the results of theoretical investigations of planetary ring dynamics to these resonant associations and evaluate their dynamical significance. We calculate the resonant torques which the satellites exert on each ring and compare them to the viscous torque which transports angular momentum outward through the ring and to the atmospheric-drag torque which causes the ring's orbit to decay. Unfortunately, the theoretical tools at our disposal are rather imprecise, especially when applied to strong resonances where linear perturbation theory is not strictly applicable. Consequently, much of the upcoming analysis is valid only to order of magnitude. To avoid a distracting stream of qualifications, we do not attempt to attach errors to our numerical estimates; they would not be very meaningful anyway.

Theoretical guidance for the calculations presented in this section is provided by Borderies, 'Goldreich, and Tremaine (1984). It is our basic source of formulas dealing with nonlinear satellite torques. Although there are several more recent papers which treat nonlinear density waves in planetary rings (Shu, Yuan, and Lissauer 1985; Shu et al. 1985; Borderies, Goldreich, and Tremaine 1985, 1986), they do not provide estimates of the torques in the limit of strong nonlinearity and realistic damping.

One of the objectives of this investigation is to determine whether the standard theory of shepherding provides an adequate explanation for the confinement of the Uranian rings. Our procedure is to assume that this is the case and to see whether this assumption leads to any contradictions.

We adopt the notation defined in Paper I in the current paper.

\section{SHEPHERDING OF NARROW RINGS}

There is extensive literature on the shepherding of narrow rings. However, nowhere does there exist an adequate summary of our current understanding, at least in a form suitable for application to observed ring-satellite systems. Accordingly, we provide a brief outline of the elements of shepherding theory in the following paragraphs. A detailed and systematic presentation of these concepts is given elsewhere (Borderies, Goldrech, and Tremaine 1986).

\section{a) Standard Theory}

The discovery of narrow rings encircling Uranus led to the prediction that each ring was shepherded by two satellites whose orbits bracket that of the ring (Goldreich and Tremaine 1979a). It was proposed that the torques exerted by the inner and outer satellite would be the sources and sinks of the angular momentum which viscous stresses, arising from interparticle collisions and differential rotation, transport outward through the ring. There are several variants of the shepherding mechanism which are distinguished by the nature of the perturbation which the satellite produces in the ring (cf. Sec. III of Paper I). Sufficiently close and massive satellites produce wakes, disturbances of limited azimuthal extent which are smooth functions of semimajor axis. Disturbances due to smaller and more distant satellites are best 
described in terms of discrete resonances. They extend around the entire circumference of the ring and are concentrated at resonant semimajor axes. A shepherd satellite may have resonances at a number of semimajor axes within the ring or just one near the ring's edge.

The imaging and occultation data obtained during the encounters of Voyagers 1 and 2 with Saturn offered the first tests of the theory of shepherding. The Encke gap and the outer edges of the $A$ and $B$ rings provided excellent examples of the shepherding of single edges. In particular, the Encke gap is carved out of the A ring by a small satellite which transfers angular momentum from its inner to its outer edge (Cuzzi and Scargle 1985). This satellite is responsible for the wakes seen in the ring material adjacent to these edges (Showalter et al. 1986). The outer edges of the A and B rings are located at the 7:6 and 2:1 resonances of the co-orbital satellites and Mimas, respectively. These edges are shepherded by discrete resonances (Porco et al. 1984). The A ring contains several sequences of discrete resonances associated with the co-orbital satellites and the F ring shepherds. Angular momentum is removed from the ring at each of these resonances; the 7:6 co-orbital resonance determines the edge location because it removes the remaining angular momentum, thereby reducing the angular-momentum luminosity to zero. The most spectacular success was the discovery of the F ring shepherds (Smith et al. 1981). However, the observation carried out by the Voyager spacecraft probably failed to locate the main component of the mass of this ring. Thus the $\mathrm{F}$ ring did not provide a precise test of the preexisting theory.

\section{b) Angular Momentum Flux Reversal}

Although the standard theory of shepherding accounts for the locations of the outer boundaries of the A and B rings and the width of the Encke division, it needs further refinement to describe their abrupt edges. Typically, these features are sharp on length scales which are some three orders of magnitude smaller than those over which the satellite torques act. Attempts to understand the origin of these sharp edges led to the theoretical discovery that satellite perturbations can produce local reversals in the direction of the viscous flux of angular momentum $F_{H}^{\mathrm{v}}$ (Borderies, Goldreich, and Tremaine 1982, 1983, 1985). This flux, which is outward in unperturbed regions of rings, is reversed within a limited interval of azimuth in strongly perturbed regions near sharp edges. The viscous luminosity of angular momentum $L_{H}^{\mathrm{v}}$, the integral of $F_{H}^{\mathrm{v}}$ over the circumference of the ring, falls smoothly to zero as the edge is approached. The length scale over which $L_{H}^{\mathrm{v}}$ diminishes is set by the length scale over which the satellite torque is applied to the ring material. What is remarkable is that the surface density $\Sigma$ maintains a nearly constant value throughout this region and only decays over a very much smaller length scale close to the edge.

The reversal of the direction of $F_{H}^{\mathrm{v}}$ is not merely a detail which must be added to the standard theory to account for the presence of sharp edges. It is an essential feature of the shepherding mechanism. In fact, it is somewhat accidental that shepherding was proposed before this point was understood. The original work on shepherding focused on the satellite torques as providing sources and sinks for $L_{H}^{\mathrm{v}}$. The viscous stress was calculated using a parametrized kinematic viscosity $v$ and the shear appropriate to an unperturbed region of the ring in which the angular velocity $n \propto a^{-3 / 2}$.
Further work revealed that these seemingly innocent simplifying assumptions placed serious limitations on the theory. The random motions of ring particles are greatly enhanced in the region where the satellite torque is exerted, thus enhancing $v$. Indeed, if the enhanced value of $v$ is combined with the Keplerian shear to calculate $L_{H}^{\mathrm{v}}$, it appears that the satellite torque cannot shepherd the ring edge. The resolution of this paradox requires using the perturbed shear in calculating $L_{H}^{\mathrm{v}}$. In the hydrodynamic (collision rate $\gg n$ ) limit, shear reversal is a necessary and sufficient condition for flux reversal. Shear reversal implies that there are limited intervals of azimuth within which the satellite perturbs the streamlines of the ring material so severely that the angular velocity increases outward. In regions where the collision rate is comparable to or smaller than $n$, shear reversal is not necessary for flux reversal; the latter may be initiated by milder perturbations of the shear.

In some narrow rings, flux reversal may play a crucial role not just close to the ring edges but throughout the interior as well. This could occur in either of two ways.

The eccentricity gradient within the ring might be so large that it would cause flux reversal. For rings which have aligned apses, like the $\epsilon$ ring, any flux reversal associated with the eccentricity gradient would be concentrated near the narrowest part of the ring. If the eccentricity gradient causes flux reversal, the viscous stress would damp the eccentricity. However, it could be maintained by the torques exerted by shepherd satellites.

Nonlinear satellite perturbations which produce flux reversal might be present at all semimajor axes across the ring. These perturbations could be wakes produced by large and close satellites or nonlinear density waves which propagate between discrete resonances associated with smaller and more distant satellites. In either case, if nonlinear satellite perturbations cover the entire range of semimajor axes across the ring, the angular-momentum luminosity within the ring might be reduced far below its unperturbed value. This possibility would lead to a new type of shepherding, one which is conceptually different from that originally proposed. Shepherd satellites would still be responsible for determining the location of the ring and for its confinement. Of course, conservation of angular momentum requires that they must also provide the sources and sinks for $L_{H}^{\mathrm{v}}$. However, the satellites would confine the ring primarily by decreasing $L_{H}^{\mathrm{v}}$ below its unperturbed value. The new shepherding mechanism requires smaller satellites than those needed for the standard shepherding mechanism. In the standard shepherding model, each satellite is assumed to have several resonances of comparable strength in the ring. If each shepherd has a single resonance at a ring edge, the required masses of the shepherds are reduced by the factor $\Delta a /\left|a-a_{\mathrm{s}}\right|$ below the value in the standard model. If shepherding proceeds by flux reversal, the satellite masses need be no larger than $\left(\Delta a /\left|a-a_{\mathrm{s}}\right|\right)^{2}$ times the values required in the standard model.

\section{c) Ring Masses}

Prior to the Voyager encounter, the only information we had concerning the masses of the Uranian rings was based on dynamical investigations of the precession of narrow, elliptic rings (Goldreich and Tremaine 1979a,b). Because the ring mass is such a crucial dynamical parameter, and because we have derived a relation for estimating the mass of the $\delta$ ring from its $m=2$ distortion, we include a brief description of 
the relevant formulas below. In the current investigations, we adopt the dynamically determined masses as preliminary estimates.

The survival of the ellipticity of narrow rings requires some mechanism to counter the tendency of the planet's quadrupole moment to cause differential precession across the ring. Goldreich and Tremaine (1979a,b) showed that the self-gravity of the ring could prevent differential precession. They argued that the pressure associated with the random motions of the ring particles would be too small to have a significant effect on the differential precession, at least if it varied smoothly on length scales comparable to the ring's width. However, they raised the possibility that large pressure gradients, associated with abrupt shock-like transitions, might play a significant role in affecting differential precession. The precise apse alignment of the elliptical inner and outer edges of the $\epsilon$ ring provides another argument for favoring a purely gravitational explanation for rigid precession. It seems unlikely that accurate apse alignment would occur if differential precession were prevented by stresses associated with particle collisions since these are inherently dissipative: that is, the same mechanism for counteracting differential precession would appear likely to lead to a nonalignment of the apses of the inner and outer ring edges. In spite of these plausible arguments, both the Voyager radio occultation data for the Uranian rings and our forthcoming dynamical results challenge the hypothesis that self-gravity is responsible for maintaining apse alignment. In particular, they suggest that this hypothesis leads us to underestimate significantly the masses of the $\alpha$ and $\beta$ rings. We return to this point in Secs. V and VI.

If self-gravity maintains apse alignment across the ring, knowledge of the planet's gravitational field and the ring's geometry would suffice to determine the ring's mass $M$. An approximate formula, derived from a simple two-streamline ring model (Borderies, Goldreich, and Tremaine 1983), gives

$$
\frac{M}{M_{\mathrm{U}}}=\frac{21 \pi}{16} J_{2}\left(\frac{R_{\mathrm{U}}}{a}\right)^{2}\left(\frac{\Delta a}{a}\right)^{3} \frac{1}{H\left(q^{2}\right)} \frac{e}{\Delta e} .
$$

Here $q \approx a \Delta e / \Delta a$, and

$$
H(x) \equiv \frac{1-(1-x)^{1 / 2}}{x(1-x)^{1 / 2}} .
$$

The masses of the elliptical rings which are adopted in this paper, $M_{\alpha} \approx 4.2 \times 10^{16} \mathrm{~g}, M_{\beta} \approx 3.8 \times 10^{16} \mathrm{~g}$, and $M_{\epsilon}$ $\approx 6.1 \times 10^{18} \mathrm{~g}$, were obtained from Eq. (1) by French et al. (1986a). Experience has shown that the masses obtained from the two-stream model are slightly larger than those obtained from multistream models.

An elliptical $m=1$ ring is a special case of a distorted circular ring in which the distortion takes the form $\delta r=a e$ $\cos m\left(\phi-\Omega_{\mathrm{p}} t\right)$. For $m=1, \Omega_{\mathrm{p}}=\dot{\omega} \ll n$, and the differential precession forced by self-gravity has only to compensate that due to the planet's quadrupole moment; hence the appearance of $J_{2}\left(R_{\mathrm{U}} / a\right)^{2} \ll 1$ in Eq. (1). For $m \neq 1, \Omega_{\mathrm{p}}$ is of order $n$ and self-gravity has to work much harder since the differential precession must compensate for the differential orbital angular velocity. This implies that only relatively narrow and dense rings can support distortions with $m \neq 1$ (Borderies et al. 1985); presumably, this is the reason why elliptical rings are so much more common than rings whose distortions correspond to $m \neq 1$. For $m \neq 1$, the two-stream model yields

$$
\frac{M}{M_{\mathrm{U}}}=\frac{3 \pi(m-1)}{8}\left(\frac{\Delta a}{a}\right)^{3} \frac{1}{H\left(q^{2}\right)} \frac{e}{\Delta e} .
$$

Equation (3) is used later in the paper to provide a mass for the $\delta$ ring.

The normal modes of distortions of narrow elliptical rings are standing density waves. Equations (1) and (3) apply to modes which have no nodes within the ring. These nodeless modes have scale lengths which are comparable to the first wavelengths of density waves. To see this, replace $M$ in Eq. (3) by $2 \pi \Sigma a \Delta a$ and solve for $\Delta a$. Setting $H\left(q^{2}\right) \Delta e / e \approx 1$ and $m \gg 1$, the resulting expression for $\Delta a$ reduces to $w_{\lambda}$ as given by Eq. (6) in Paper I.

\section{SHEPHERDING OF THE $\epsilon$ RING}

\section{a) Torques on the $\epsilon$ Ring}

Kinematical evidence suggesting that $1986 \mathrm{U} 7$ and 1986 U 8 are shepherding the $\epsilon$ ring was presented in Paper I. We turn now to an assessment of the dynamical evidence.

In general, angular momentum flows outward through an unperturbed disk due to the viscous torque, $T_{\mathrm{v}} \equiv L_{H}^{\mathrm{v}}$, arising from particle collisions in the presence of differential rotation. An isolated narrow ring will spread unless angular momentum is supplied at its inner edge and removed at its outer edge. The $\epsilon$ ring is not isolated since it is perturbed by the resonances associated with $1986 \mathrm{U} 7$ and 1986U8. However, the strongest resonances are located at the ring's edges, which suggests that the interior region may not be strongly disturbed. In order to apply the standard theory of shepherding to the $\epsilon$ ring, we assume that $L_{H}^{\mathrm{v}}$ is unperturbed in its interior. However, we note that the mean eccentricity gradient across this ring is relatively large, $q \approx 0.67$, and that the torque at the 47:49 resonance of 1986U7 near the ring's center is almost as large as the first-order torques on the ring's edges. Thus, it is not impossible that flux reversal occurs in the ring's interior. Nevertheless, we proceed to test the standard shepherding model by assuming that 1986U7 and 1986U8 exert torques which equal $\pm T_{\mathrm{v}}$, the unperturbed viscous torque at the ring's inner and outer edges.

To evaluate $T_{\mathrm{v}}$ and $T_{\mathrm{s}}$, the masses of the ring and the shepherd satellites must be known. We have already discussed the manner in which ring masses are deduced. We adopt $M_{\epsilon}=6.1 \times 10^{18} \mathrm{~g}$, which corresponds to a mean surface density $\Sigma=33 \mathrm{~g} \mathrm{~cm}^{-2}$. The mass of each shepherd was calculated in Paper I: $M_{7} \approx 5 \times 10^{19} \mathrm{~g} ; M_{8} \approx 9 \times 10^{19} \mathrm{~g}$. The uncertainties are about a factor of 2 . All uncertainties considered, we conclude that the satellites are more massive than the ring.

The transfer of angular momentum from 1986U7 to the $\epsilon$ ring and from the $\epsilon$ ring to 1986U8 causes the separation $a_{8}-a_{7}$ between the satellites to increase. These satellites do not appear to be in stable resonances with any larger satellites. Thus a firm upper bound to the total angular momentum $\Delta L_{\epsilon}$ which has been passed through the $\epsilon$ ring is given by

$$
\begin{aligned}
\Delta L_{\epsilon} & \leqslant \frac{1}{2} \sqrt{G M_{\mathrm{U}}}\left(\frac{M_{7} M_{8}\left(a_{8}-a_{7}\right)}{M_{7} \sqrt{a_{8}}+M_{8} \sqrt{a_{7}}}\right) \\
& =7 \times 10^{33} \mathrm{~g} \mathrm{~cm}^{2} \mathrm{~s}^{-1} .
\end{aligned}
$$

We denote the lifetime of this system by

$$
t_{\epsilon}=f t_{\mathrm{ss}} \text {, }
$$

where $f \leqslant 1$ and $t_{\mathrm{ss}}=4.6 \times 10^{9} \mathrm{yr}$ is the age of the solar sys- 
tem. The rate of increase in the separation $a_{8}-a_{7}$ is likely to have decreased as the satellites moved away from each other and the ring. Hence, the current viscous luminosity of angular momentum in the $\epsilon$ ring must satisfy

$$
L_{H}^{\mathrm{v}} \equiv T_{\mathrm{v}} \equiv\left(\frac{\Delta L_{\epsilon}}{t_{\epsilon}}\right) \leqslant 5 \times 10^{16} f^{-1} \mathrm{erg} .
$$

The viscous torque may be expressed as

$$
T_{\mathrm{v}} \approx 3 \pi \Sigma v n a^{2} \text {, }
$$

where $v$ is the kinematic viscosity. From Eqs. (6) and (7), we deduce that

$$
v \lesssim 0.03 f^{-1} \mathrm{~cm}^{2} \mathrm{~s}^{-1} \text {. }
$$

It is revealing to compare this number to the minimum viscosity the $\epsilon$ ring could have given its surface mass density and optical depth. The latter would be achieved if the particles were close packed and the ring material behaved more like an incompressible liquid than a gas. The minimum viscosity is given by

$$
v_{\mathrm{m}} \approx n\left(\frac{\Sigma}{\rho}\right)^{2},
$$

where $\rho$ is the bulk density of the ring material (Borderies et al. 1985). Evaluating $v_{\mathrm{m}}$ for parameter values appropriate to the $\epsilon$ ring, $\Sigma=33 \mathrm{~g} \mathrm{~cm}^{-2}$ and $\rho=1.4 \mathrm{~g} \mathrm{~cm}^{-3}$, we obtain $v_{\mathrm{m}} \approx 0.1 \mathrm{~cm}^{2} \mathrm{~s}^{-1}$, slightly larger than the upper limit on $v$ for $f=1$.

The standard expressions for the satellite torques at firstand second-order Lindblad resonances and at first-order corotation resonances are quoted below in a form appropriate for $m \gg 1$.

$$
\begin{aligned}
& T_{1}^{\mathrm{L}}=\mp 8.6 m^{2}\left(\frac{M_{\mathrm{s}}}{M_{\mathrm{U}}}\right)^{2} \Sigma n^{2} a^{4}, \\
& T_{2}^{\mathrm{L}}=\mp 3.0 e_{\mathrm{s}}^{2} m^{4}\left(\frac{M_{\mathrm{s}}}{M_{\mathrm{U}}}\right)^{2} \Sigma n^{2} a^{4}, \\
& T_{1}^{\mathrm{C}}=-13 e_{\mathrm{s}}^{2} m^{3}\left(\frac{M_{\mathrm{s}}}{\mathrm{M}_{\mathrm{U}}}\right)^{2} \Sigma n^{2} a^{4}\left[1+\frac{2}{3} \frac{a}{\Sigma} \frac{d \Sigma}{d a}\right] .
\end{aligned}
$$

The upper and lower signs on the Lindblad torques apply to ILR and OLR, respectively.

The familiar Lindblad resonances do not exist in an eccentric ring. They are replaced by more complex resonances, referred to as eccentric resonances. The resonances in an eccentric ring are classified and described in Paper I. The standard torque at an $n$ th-order eccentric resonance may be decomposed into two parts, one similar to that at an $n$ thorder Lindblad resonance and another similar to that at an $n$ th-order corotation resonance. Thus the torque at a firstorder eccentric resonance is given by the sum of $T_{1}^{\mathrm{L}}$ and $T_{1}^{\mathrm{C}}$, with $e_{\mathrm{s}}$ replaced by $e$. As mentioned previously in Paper I, there are two distinct second-order eccentric resonances characterized by $k=1, q=1$ and by $k=0, q=2$. The Lindblad portion of the second-order torque is $T_{2}^{\mathrm{L}}$ at the former and $T_{2}^{\mathrm{L}}$, with $e_{\mathrm{s}}$ replaced by $e$, at the latter. The second-order corotation torques are too small to be of interest here.

Each torque arises from a second-order interaction between the potential and the disturbance it excites in the disk; if the disk disturbance is linear, the torque is proportional to the square of the forcing potential. For example, the standard expressions for the Lindblad torque apply if the density wave excited at the Lindblad resonance is linear in its first few wavelengths. There is no precise theoretical expression analogous to Eq. (10) for the nonlinear Lindblad torque at a first-order eccentric resonance. However, some relevant semiquantitative results are available (Borderies, Goldreich, and Tremaine 1984). The transition from linear to nonlinear behavior occurs at a critical torque,

$$
T_{1, \mathrm{c}}^{\mathrm{L}} \approx \frac{\pi^{2} \Sigma^{3} n^{2} a^{8}}{6 M_{\mathrm{U}}^{2}} .
$$

In the nonlinear regime, the density wave saturates so its amplitude is independent of that of the forcing potential. Thus the nonlinear torque is directly proportional to the forcing potential and, for a first-order eccentric resonance, reads:

$$
T_{1, \mathrm{nl}}^{\mathrm{L}} \approx \mp \frac{3.7 m M_{\mathrm{s}} \Sigma^{2} n^{2} a^{6}}{M_{\mathrm{U}}^{2}} .
$$

The expression for the nonlinear torque applies if the azimuthally averaged surface density is constant within the region containing at least a few waves of the density wave train launched at the resonance. Shu, Yuan, and Lissauer (1985) have shown that nonlinear effects increase the wavelengths of density waves. The nonlinear wavelength increase has been neglected in the derivation of Eq. (14). This is not a serious oversight because the damping associated with particle collisions limits the nonlinearities of density waves in planetary rings to modest values. The principal consequence of the nonlinear wavelength increase is to increase the nonlinear torque given in Eq. (14) by a factor of order unity.

There is an important relation between the critical torque $T_{1, \mathrm{c}}^{\mathrm{L}}$ given by Eq. (13) and the minimum viscous torque $T_{\mathrm{v}_{\mathrm{m}}}$, which follows from substituting Eq. (9) into Eq. (7). A short computation reveals that

$$
T_{\mathrm{v}_{\mathrm{m}}} \approx 10^{2}\left(\frac{R_{\mathrm{U}}}{a}\right)^{6}\left(\frac{\rho_{\mathrm{U}}}{\rho}\right)^{2} T_{1, \mathrm{c}}^{\mathrm{L}} \approx T_{1, \mathrm{c}}^{\mathrm{L}},
$$

where the final numerical evaluation is for parameters appropriate to the rings of Uranus. Equation (15) implies that any satellite torque large enough to shepherd a ring edge must exceed $T_{1, \mathrm{c}}^{\mathrm{L}}$.

The expressions for the linear and nonlinear torques at the strongest resonances of 1986U7 and 1986U8 in the $\epsilon$ ring have been evaluated using the planet and satellite parameters given in Table I of Paper I, the geometry appropriate to the present satellite-ring configuration and the mean surface mass density. They are listed in Table I. We have just proved that a nonlinear Lindblad torque is required to maintain a ring edge. For the specific cases of the $\epsilon$ ring edge resonances, we find that the linear Lindblad torques are about a factor 10 greater than the critical torque $T_{\mathrm{c}} \approx 2 \times 10^{17} \mathrm{erg}$. Even the nonlinear Lindblad torques listed in Table I overestimate the actual satellite torque $T_{\mathrm{s}}$ because $\Sigma$ drops abruptly to zero at the sharp edges of the ring. We note that the critical torque is insensitive to resonance order and that the Lindblad torque at the 47:49 eccentric resonance of 1986U7, which is located close to the center of the $\epsilon$ ring, is comparable to the critical value. Thus we would expect that the resonance is the site of a nonlinear disturbance in the ring. If the ring had $q=0$, this disturbance would be a nonlinear density wave similar to those discovered in Saturn's rings. Although there is no theory to describe linear, let alone nonlinear, disturbances in a ring for which $q \neq 0$, and $q_{\epsilon} \approx 0.67$, it is plausible that a distorted density wave could propagate through the $\epsilon$ ring. At present, there is no unambiguous observational confirma- 
TABLE I. Torques.

\begin{tabular}{|c|c|c|}
\hline Type & $\begin{array}{l}\text { Location } \\
(\mathrm{km})\end{array}$ & $\begin{array}{l}\text { Torque }^{a} \\
\text { (erg) }\end{array}$ \\
\hline $\begin{array}{l}\text { 1st order Lindblad } \\
\text { 1986U8 14:13 IER } \\
\text { 1986U7 24:25 OER } \\
\text { 1986U7 23:24 OER }\end{array}$ & 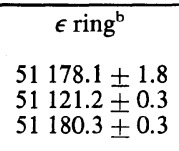 & $\begin{array}{l}-2 \times 10^{18}\left(-5 \times 10^{17}\right) \\
+2 \times 10^{18}\left(+5 \times 10^{17}\right) \\
+2 \times 10^{18}\left(+5 \times 10^{17}\right)\end{array}$ \\
\hline $\begin{array}{l}\text { 2nd order Lindblad } \\
\text { 1986U8 28:26 IER } \\
\text { 1986U7 47:49 OER }\end{array}$ & $\begin{array}{ll}51 & 177.8 \pm 1.8 \\
51 & 150.1 \pm 0.3\end{array}$ & $\begin{array}{l}-2 \times 10^{17} \\
+3 \times 10^{17}\end{array}$ \\
\hline $\begin{array}{l}\text { 1st order corotation } \\
\text { 1986U8 } 14: 13 \mathrm{CR}^{\mathrm{c}} \\
\text { Viscous }^{\mathrm{d}} \\
\text { Atmospheric } \\
\text { Critical }\end{array}$ & $\begin{array}{c}51177.5 \pm 1.8 \\
- \\
-\end{array}$ & $\begin{aligned} & -4 \times 10^{15} \\
\leqslant & +5 \times 10^{16} f^{-1} \\
- & 1 \times 10^{17} \\
& 2 \times 10^{17}\end{aligned}$ \\
\hline $\begin{array}{l}\text { 1st order Lindblad } \\
\text { 1986U7 23:22 IER } \\
\text { Atmospheric } \\
\text { Critical }\end{array}$ & $\begin{array}{c}\delta \text { ring }^{\mathrm{b}} \\
48302.5 \pm 0.3 \\
-\end{array}$ & $\begin{array}{l}-5 \times 10^{17}\left(-4 \times 10^{16}\right) \\
-3 \times 10^{16} \\
\quad 3 \times 10^{15}\end{array}$ \\
\hline $\begin{array}{l}\text { 1st order Lindblad } \\
\text { 1986U8 6:5 IER } \\
\text { Atmospheric } \\
\text { Critical }\end{array}$ & $\begin{array}{c}\gamma \text { ring }^{\mathrm{b}} \\
47625.7 \pm 1.8 \\
-\end{array}$ & $\begin{array}{l}-1 \times 10^{17}\left(-2 \times 10^{16}\right) \\
-3 \times 10^{16} \\
\quad 3 \times 10^{15}\end{array}$ \\
\hline
\end{tabular}

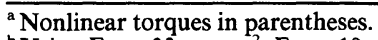

${ }^{\mathrm{b}}$ Using $\Sigma_{\epsilon}=33 \mathrm{~g} \mathrm{~cm}^{-2}, \Sigma_{\delta}=10 \mathrm{~g} \mathrm{~cm}^{-2}$, and $\Sigma_{\gamma}=10 \mathrm{~g} \mathrm{~cm}^{-2}$.

${ }^{c}$ Calculated for $d \Sigma / d a=0$.

${ }^{\mathrm{d}}$ See the text for definition of $f$.

tion of any disturbance associated with this resonance. However, we have noted the proximity of this resonance to a wave-like feature pointed out by the photopolarimeter team in the PPS $\sigma$ Sag ingress occultation scan of the $\epsilon$ ring (see Fig. 4, Paper I).

The reduction of the corotation torque due to saturation is particularly severe; viscous diffusion is too slow to flush out the particles whose semimajor axes librate about the resonance value. A quantitative measure of the saturation of the corotation torque is obtained following the procedure outlined in Goldreich and Tremaine (1981). We define the dimensionless saturation parameter $S$ to be the ratio of the viscous diffusion time across the region of librating orbits to the libration period. Thus

$$
S \approx \frac{\omega_{1}\left(\Delta a_{1}\right)^{2}}{v} .
$$

Next, we cast $S$ in a form appropriate for first-order corotation torques. For definiteness, we specialize to the case $k=1, q=0$. Since the orbital eccentricities of the $\epsilon$ ring and the outer shepherd are comparable, the numerical values for $S$ are the same for all the important first-order corotation torques in the $\epsilon$ ring. We substitute the expressions

$$
\omega_{1} \approx m^{3 / 2} e_{\mathrm{s}}^{1 / 2}\left(\frac{M_{\mathrm{s}}}{M_{\mathrm{U}}}\right)^{1 / 2} n
$$

for the frequency of small librations about resonance and

$$
\Delta a_{1} \approx m^{1 / 2} e_{\mathrm{s}}^{1 / 2}\left(\frac{M_{\mathrm{s}}}{\mathrm{M}_{\mathrm{U}}}\right)^{1 / 2} a
$$

for the semimajor-axis variation across the region of librating orbits into Eq. (16) and arrive at

$$
S \approx m^{5 / 2} e_{\mathrm{s}}^{3 / 2}\left(\frac{M_{\mathrm{s}}}{M_{\mathrm{U}}}\right)^{3 / 2} \frac{n a^{2}}{v} .
$$

Evaluating $S$ for parameters appropriate to the first-order corotation torques in the $\epsilon$ ring, we find $S \approx 10^{2} \mathrm{~cm}^{2} \mathrm{~s}^{-1} / v$. Thus $S \gg 1$ for the values of $v=0(1) \mathrm{cm}^{2} \mathrm{~s}^{-1}$ which we think characterize the $\epsilon$ ring. For this reason, we disregard the corotation torques in this paper.

The discovery of a distended upper atmosphere (Broadfoot et al. 1986) was a surprising result of the Voyager encounter with Uranus. From modeling the solar and $\gamma$ Pegasus occultation light curves, the exosphere was found to have a temperature of $750 \pm 100 \mathrm{~K}$ above $27700 \mathrm{~km}$ and a hydrogen number density of $1 \times 10^{8} \mathrm{~cm}^{-3}$ at $r=27930 \mathrm{~km}$. These results were combined to yield

$$
N_{\mathrm{H}}(r)=7 \times 10^{-6} e^{32.4 / r} \mathrm{~cm}^{-3},
$$

where $r$ is given in units of $R_{\mathrm{U}}$. Broadfoot et al. (1986) also detected Ly $\alpha$ emission out to several $R_{\mathrm{U}}$ in excess of that expected from resonant scattering by the thermal distribution of atomic hydrogen assumed in Eq. (20). They have interpreted this excess emission as evidence for additional $\mathbf{H}$ excitation or a nonthermal distribution of $\mathrm{H}$. However, the $\mathrm{H}$ number density associated with this excess emission is believed to be negligible in comparison to that of the thermal population, even at the distance of the $\epsilon$ ring (Hunten 1986). Equation (20) yields $N_{\mathrm{H}}\left(a_{\epsilon}\right)=115 \pm 60 \mathrm{~cm}^{-3}$; the uncertainty arises from the $\pm 100 \mathrm{~K}$ uncertainty in the exospheric temperature.

The hydrogen atoms collide with the particles of the $\epsilon$ ring exerting a torque

$$
T_{\mathrm{d}} \approx-4 \pi N_{\mathrm{H}}(a) m_{\mathrm{H}} v_{\mathrm{T}} n a^{3} \Delta a \approx-1 \times 10^{17} \mathrm{erg},
$$

where $v_{\mathrm{T}}$ is the average thermal speed of the hydrogen atoms normal to the ring plane. Equation (21) takes into account that the angular velocity of the hydrogen atoms about the planet is much smaller than $n$ and that the mean optical depth of the $\epsilon$ ring is somewhat greater than unity. We note that, for $f=1, T_{\mathrm{d}}$ is comparable to the upper limit on $T_{\mathrm{v}}$. 


\section{b) Consequences of Shepherding of the $\epsilon$ Ring}

In Paper I we presented two independent lines of evidence which indicate that the absolute radius scale of the Uranian rings should be reduced. One of these is the alignment of the first-order eccentric resonances, the 24:25 OER of 1986U7 and the 14:13 IER of 1986U8, with the inner and outer edges of the $\epsilon$ ring. Furthermore, we have shown in the previous section that the torques exerted by the satellites at these resonances have the correct signs and adequate magnitude to supply at the inner edge, and remove at the outer edge, the viscous luminosity of angular momentum which flows through the ring. Thus we feel justified in asserting that 1986U 7 and 1987U8 are shepherding the $\epsilon$ ring and that the ring radii derived from ground-based occultation data must be reduced. Having accepted that 1986U7 and 1986U8 are the $\epsilon$ ring shepherds, there are a number of additional inferences we can draw.

We have demonstrated that if the $\epsilon$ ring and its shepherds have been around for a long time, i.e., $f=1$, the ring particles must be fairly close packed. Tyler et al. (1986) deduce from the azimuthal variation of the ring's optical depth that the particles are well separated. The situation thus merits further consideration. A plausible resolution is that uncertainties in the values of the satellite masses and the coefficient in the nonlinear torque formula, among other things, are sufficient to resolve this problem. Nevertheless, it is illuminating to explore other possibilities.

If $f \ll 1$, the upper bound on $T_{\mathrm{v}}$ is relaxed. However, $T_{\mathrm{v}}=\left|T_{\mathrm{s}}\right|$ is a requirement of the shepherding hypothesis. The linear and nonlinear torques listed in Table I are upper bounds to $\left|T_{\mathrm{s}}\right|$. Using the linear torques, which provide the more generous upper limits to $\left|T_{\mathrm{s}}\right|$, we find that $v \leqslant 10 v_{\mathrm{m}}$. For rings whose particles are well separated, we can express $v$ as a function of the thickness $h$ and optical depth $\tau$ as $v \approx n h^{2} \tau /\left(1+\tau^{2}\right)$ (Goldreich and Tremaine 1978). Thus $h \lesssim(\Sigma / \rho)$; apparently, most of the material in the $\epsilon$ ring is confined to a layer which is no more than a couple of meters thick, whatever its age. It is more difficult to obtain a lower bound on $f$. The satellites could have receded from much closer together to their present separation in $\sim 5 \times 10^{7} \mathrm{yr}$ if $v$ is close to its maximum possible value. However, we cannot exclude the possibility that they were formed near their current positions. A more secure but less restrictive limit comes from the viscous spreading time of the $\epsilon$ ring. The ring clearly fills a region of mean width $\Delta a_{\epsilon}=60 \mathrm{~km}$ between the confining resonances. The viscous diffusion time across this region is $t_{\mathrm{v}} \approx\left(\Delta a_{\epsilon}\right)^{2} / v \gtrsim 10^{6} \mathrm{yr}$, where the lower limit to $t_{\mathrm{v}}$ corresponds to the upper limit to $v$.

Heretofore, we have adopted the value for the ring mass which follows from the hypothesis that self-gravity maintains a uniform apse precession rate across the ring. Now, we adopt this assumption and examine the constraints that the shepherding hypothesis places on the ring mass. Shepherding requires $\left|T_{\mathrm{s}}\right| \geqslant\left|T_{\mathrm{d}}\right|$ and thus sets a lower limit on $M_{\epsilon}$ because $T_{\mathrm{d}}$ is independent of $m_{\epsilon}$, whereas $T_{1, \mathrm{nl}}^{\mathrm{L}}$ is directly proportional to $M_{\epsilon}^{2}$. From the numerical values listed in Table I, we see that $M_{\epsilon} \gtrsim 10^{18} \mathrm{~g}$. Shepherding also requires $\left|T_{1, \mathrm{n} l}^{\mathrm{L}}\right| \geqslant T_{\mathrm{v}}$ and thus sets an upper limit on $M_{\epsilon}$ since $T_{1, \mathrm{nl}}^{\mathrm{L}} \propto M_{\epsilon}^{2}$ and the minimum value of $T_{\mathrm{v}} \propto v_{\mathrm{m}} \Sigma \propto M_{\epsilon}^{3}$. Again appealing to the numerical values in Table I, we find $M_{\epsilon} \leqslant 3 \times 10^{19} \mathrm{~g}$. Thus we conclude that $10^{18} \mathrm{~g} \leqslant M_{\epsilon}$ $\leqslant 3 \times 10^{19} \mathrm{~g}$. These formal limits are probably a bit too restrictive considering the substantial uncertainties in the nu- merical values of the coefficients and observationally determined input parameters which enter the expressions for $T_{\mathrm{d}}, T_{\mathrm{s}}$ and the minimum value of $T_{\mathrm{v}}$. However, they bracket the nominal value of $M_{\epsilon} \approx 6 \times 10^{18} \mathrm{~g}$ and rule out values widely different from it.

For $f=1$ the value of $\left|T_{\mathrm{d}}\right|$ is uncomfortably close to the upper limit to $T_{\mathrm{v}}$. However, although we know the correct functional forms for $T_{\mathrm{d}}$ and $T_{\mathrm{v}}$, our knowledge of some of the numerical coefficients and of certain observationally determined input parameters is not adequate to allow us to draw any firm conclusions from the near equality of these two torques. All we can say is that $\left|T_{\mathrm{d}}\right|<T_{\mathrm{v}}$; otherwise, the outer edge of the $\epsilon$ ring would not press against the 14:13 IER of $1986 \mathrm{U} 8$. We shall see later in the paper that $T_{\mathrm{d}}$ poses a very serious constraint on the shepherding of some of the other Uranian rings.

\section{SHEPHERDING OF THE $\delta$ AND $\gamma$ RINGS}

Before computing the mass of the $\delta$ ring from Eq. (3), we must obtain values for the parameters $e, \Delta a$, and $\Delta e$. The value $e \approx 6 \times 10^{-5}$ follows immediately from the fit to the $m=2$ distortion found by French et al. (1986b), which has $a e \approx 3 \mathrm{~km}$. To obtain $\Delta a$ and $\Delta e$, we use the data in French $e t$ al. (1986a), which shows that the ring width $W_{\delta}$ satisfies 2 $\mathrm{km} \leqslant W_{\delta} \leqslant 8 \mathrm{~km}$. Furthermore, there is some rather weak evidence for a width-radius relation, a correlation (positive in this case) between width and radius, as would be expected for a distortion of any $m$ (see their Fig. 8). The relations for the minimum and maximum width, $\Delta a(1-q)$ and $\Delta a(1+q)$, yield $\Delta a \approx 5 \mathrm{~km}, q \approx 0.6$ and $\Delta e \approx 6 \times 10^{-5}$. Note that $\Delta e \approx e$. Substituting these values into Eq. (3) yields $M_{\delta} \approx 1.6 \times 10^{17} \mathrm{~g}$, which corresponds to a mean surface density $\Sigma_{\delta} \approx 10 \mathrm{~g} \mathrm{~cm}^{-2}$. Thus the surface density of the $\delta$ ring is intermediate between the low values for the $\alpha$ and $\beta$ rings and the high value for the $\epsilon$ ring.

The discussion of the shepherding of the $\delta$ ring parallels that for the $\epsilon$ ring, the chief difference arising because, in this case, the inner shepherd, which we name satellite $x$, has not been detected. Suppose that satellite $x$ is located midway between the $\gamma$ and $\delta$ rings, $\sim 330 \mathrm{~km}$ inside the orbit of the $\delta$ ring. Its first-order eccentric resonances in the vicinity of the ring would have $m \sim 100$ and be separated by $\sim 3.5 \mathrm{~km}$, which is less than $\Delta a_{\delta} \approx 5 \mathrm{~km}$, the variation in semimajor axis across the $\delta$ ring. Thus we might expect satellite $x$ to have more than one first-order eccentric resonance in the $\delta$ ring. We obtain a lower limit $M_{x}$ from setting $T_{1, \mathrm{nl}}^{\mathrm{L}}$ as given by Eq. (14) equal to $T_{\mathrm{v}_{\mathrm{m}}}$ as given in Eq. (15). This procedure yields $M_{x} \gtrsim 10^{18} \mathrm{~g}$, which corresponds to $R \gtrsim 6 \mathrm{~km}$.

A slightly more restrictive lower bound on the mass and size of satellite $x$ can be obtained from the requirement that its torque on the ring be sufficient to balance that due to atmospheric drag. For the $\delta$ ring, $T_{\mathrm{d}} \approx 2.5 \times 10^{16} \mathrm{erg}$, which is approximately 10 times larger than $T_{\mathrm{v}_{\mathrm{m}}}$. Setting $T_{\mathrm{d}}$ equal to $T_{1, \mathrm{nl}}^{\mathrm{L}}$ yields a satellite radius of $R_{x} \gtrsim 10 \mathrm{~km}$. Satellites of this size would be just at the limit of detectability claimed by the Voyager Imaging Team. Similar arguments may be applied to the $\gamma$ ring. A satellite of radius $R_{x} \gtrsim 11 \mathrm{~km}$, positioned midway between the $\gamma$ and $\eta$ rings, would be capable of balancing the atmospheric drag for a surface density $\Sigma_{\gamma} \approx 10 \mathrm{~g} \mathrm{~cm}^{-2}$. Given the large optical depths measured by the Voyager occultation experiments for the $\gamma$ ring (Lane $e t$ al. 1986; Tyler et al. 1986), such a surface density is not unreasonable. 


\section{SHEPHERDING OF THE $\alpha$ AND $\beta$ RINGS}

The calculated surface densities, $\Sigma_{a} \approx 2.0 \mathrm{~g} \mathrm{~cm}^{-2}$ and $\Sigma_{\beta} \approx 1.5 \mathrm{~g} \mathrm{~cm}^{-2}$, are much smaller than $\Sigma_{\epsilon} \approx 33 \mathrm{~g} \mathrm{~cm}^{-2}$. Furthermore, $a_{\alpha}$ and $a_{\beta}$ are smaller than $a_{\epsilon}$. Both of these factors enhance the importance of the atmospheric-drag torque relative to the torques produced by shepherd satellites for these rings. The problems this poses for the shepherding of each ring are essentially identical. We arbitrarily focus on the $\beta$ ring as a concrete example.

Suppose the inner $\beta$ ring shepherd, again denoted as satellite $x$, were located approximately midway between the $\alpha$ and $\beta$ rings, $\approx 470 \mathrm{~km}$ inside the $\beta$ ring. Its first-order eccentric resonances would have $m \approx 65$ and be spaced by $\approx 6.8$ $\mathrm{km}$ in the vicinity of the ring. Thus we might expect the inner shepherd to have two such resonances in the ring. We use Eq. (14) to obtain

$$
T_{\mathrm{s}} \lesssim 2 T_{1, \mathrm{nl}}^{\mathrm{L}} \approx 4 \times 10^{15}\left(\frac{R_{x}}{10 \mathrm{~km}}\right)^{3} \mathrm{erg},
$$

for the upper bound to the total torque which the inner shepherd could exert on the $\beta$ ring. For $R_{x}$ near its upper limit of $10 \mathrm{~km}, T_{\mathrm{s}}$ could be substantially larger than the minimum viscous torque,

$$
T_{\mathrm{v}_{\mathrm{m}}} \approx 1 \times 10^{13} \mathrm{erg},
$$

which follows from Eqs. (7) and (9). However, $T_{\mathrm{s}}$ cannot be anywhere near as large as the magnitude of the atmospheric-drag torque,

$$
T_{\mathrm{d}} \approx-1 \times 10^{17} \mathrm{erg},
$$

which is predicted by Eqs. (21) and (20).

We have not been able to resolve this problem with the shepherding of the $\alpha$ and $\beta$ rings. The source of the difficulty is the small surface density which is implied by the self-gravity hypothesis for apse locking. The relatively high $s$ band opacities of these rings, which were measured by the Voyager Radio Science Team (Tyler et al. 1986), provide independent evidence that the dynamical masses may be too small since they imply that the rings are filled with particles which are at least several centimeters in size. In fact, the surface densities could be raised by up to an order of magnitude without imperiling the shepherding requirement $T_{\mathrm{s}}>T_{\mathrm{v}}$, at least if the viscous torque is close to its minimum value. This would also serve to increase $T_{1, \mathrm{nl}}^{\mathrm{L}}$ by two orders of magnitude, thus making it greater than $\left|T_{\mathrm{d}}\right|$. However, we would then be forced to invent another explanation for the rigid precession of these elliptical rings. The most obvious possibility, already alluded to in Sec. II, is that the rings contain shocks across which there is a sharp rise in the stresses due to particle collisions. The repulsion due to the enhanced pressure might compensate almost all of the attraction due to self-gravity and thus prevent the outer part of the ring from precessing more rapidly than the inner portion. However, as for the $\epsilon$ ring, the close apse alignment of the inner and outer edges of the $\alpha$ and $\beta$ rings seems difficult to reconcile with pressure gradients playing a significant role in the prevention of differential precession.

\section{DISCUSSION AND CONCLUSIONS}

There is strong evidence, both kinematical and dynamical, to support the hypothesis that 1986U7 and 1986U8 are shepherding the $\epsilon$ ring. Furthermore, there is no compelling reason to believe that the standard theory of shepherding needs modification to explain the confinement of this ring. However, the values of the maximum shepherd torques which we have calculated are disconcertingly close to the minimum viscous torque and the atmospheric torque. Perhaps more generous margins would result from an accurate calculation of the nonlinear torque at a ring edge or from a more accurate determination of the masses of the shepherds. If we accept the standard theory, the dynamical evidence implies that the $\epsilon$ ring is rather flat, at most a few meters in thickness, and that its mass does not differ greatly from the value determined from the hypothesis that self-gravity is responsible for maintaining its apse alignment. Another possibility is that flux reversal, associated with either the eccentricity gradient or nonlinear density waves, reduces $T_{\mathrm{v}}$ far below its unperturbed value, and this is the principal means by which $1986 \mathrm{U} 7$ and $1986 \mathrm{U} 8$ confine the $\epsilon$ ring.

We close on an unsettling note. The torque due to gas drag associated with the distended hydrogen atmosphere of Uranus $T_{\mathrm{d}}$ is probably not of great significance to the shepherding of the $\epsilon$ and $\delta$ rings. This comment is based on the observed morphologies of the rings and not on the computed magnitude of $T_{\mathrm{d}}$, which in most cases we found to be comparable to $T_{\mathrm{v}}$. A problem arises, however, when we extend our shepherding arguments to the $\alpha$ and $\beta$ rings, for which the self-gravity hypothesis yields small surface densities. We estimate $T_{\mathrm{d}}$ for each ring from its surface area and the atmospheric density at its orbit. An upper limit on $T_{\mathrm{s}}$ is set from the upper bound on the sizes of potential shepherd satellites form the Voyager imaging results, the surface density of the ring, and reasonable estimates of the separation between the shepherd satellites and the ring. The result of this exercise is that $\left|T_{\mathrm{s}}\right| \ll\left|T_{\mathrm{d}}\right|$, in contradiction to the requirements of the shepherding process. This problem may be resolved by discarding the masses predicted by the self-gravity hypothesis and adopting much larger values. This would also alleviate the problem which the small surface densities pose for providing the large $s$ band radio opacities. However, we are then left without a theoretical explanation for the rigid precession of these rings, and the most plausible hypothesis, that shocks are involved, may lead to difficulties with their apse alignment.

We thank Dr. Scott Tremaine for fruitful discussions and for the hospitality extended to us by him and the staff at the Canadian Institute for Theoretical Astrophysics at the University of Toronto, where part of this research was conducted. This work was supported under JPL Contract No. 954057, NASA grant NGL-05-002-003, and NSF grant AST-8313725. 


\section{REFERENCES}

Borderies, N., Goldreich, P., and Tremaine, S. (1982). Nature 299, 209. Borderies, N., Goldreich, P., and Tremaine, S. (1983). Icarus 55, 124. I Borderies, N., Goldreich, P., and Tremaine, S. (1984). In Planetary Rings, edited by R. Greenberg and A. Brahic (University of Arizona, Tucson), pgs. 713-734.

Borderies, N., Goldreich, P., and Tremaine, S. (1985). Icarus 63, 406. Borderies, N., Goldreich, P., and Tremaine, S. (1986). Icarus 68, 522. Broadfoot, A. L., et al. (1986). Science 233, 74.

Cuzzi, J. N., and Scargle, J. D. (1985). Astrophys. J. 292, 276.

French, R. G., Elliot, J. L., and Levine, S. E. (1986a). Icarus 67, 134 French, R. G., Kangas, J. A., and Elliot, J. L. (1986b). Science 231, 480. Goldreich, P., and Tremaine, S. (1978). Icarus 34, 227.

Goldreich, P., and Tremaine, S. (1979a). Nature 277, 97.

Goldreich, P., and Tremaine, S. (1979b). Astron. J. 84, 1638.
Goldreich, P., and Tremaine, S. (1981). Astrophys. J. 243, 1062. Hunten, D. (1986). Private communication.

Porco, C., Danielson, G. E., Goldreich, P., Holberg, J. B., and Lane, A. L. (1984). Icarus 60, 17.

Porco, C. C., and Goldreich, P.`(1987). Astron. J. 93, 724 (Paper I).

Lane, A. L., et al. (1986). Science 233, 65.

Showalter, M. R., Cuzzi, J. N., Marouf, E. A., and Esposito, L. W. (1986). Icarus 66, 297

Shu, F., Yuan, C., and Lissauer, J. J. (1985). Astrophys. J. 291, 356.

Shu, F., Dones, L. Lissauer, J. J., Yuan C., and Cuzzi, J. N. (1985). Astrophys. J. 299, 542.

Smith, B. A., et al. (1981). Science 212, 163.

Smith, B. A., et al. (1986). Science 233, 43.

Tyler, G. L., et al. (1986). Science 233, 79. 